\title{
The impact of diet liberalization on bowel preparation for colonoscopy
}

\section{(잉 $\circledast$}

\section{Authors}

James Walter ${ }^{1}$, Gloria Francis², Rebecca Matro², Ramalinga Kedika², Rachael Grosso ${ }^{2}$, Scott W. Keith ${ }^{3}$, David Kastenberg ${ }^{2}$

Institutions

1 Department of Gastroenterology, Cooper University, Mount Laurel, New Jersey, United States

2 Department of Gastroenterology and Hepatology, Thomas Jefferson University Hospitals, Philadelphia, Pennsylvania, United States

3 Pharmacology and Experimental Therapeutics, Thomas Jefferson University, Philadelphia, Pennsylvania, United States

submitted 8.11 .2015

accepted after revision $\quad 30.12 .2016$

Bibliography

DOI http://dx.doi.org/10.1055/s-0043-101694 |

Endoscopy International Open 2017; 05: E253-E260

(c) Georg Thieme Verlag KG Stuttgart · New York

ISSN 2364-3722

\section{Corresponding author}

James Walter, MD, 501 Fellowship Road, Suite 101, Mount Laurel, NJ, United States 08054

Phone: +1-856-642-2133

jwwalter@gmail.com

\section{ABSTRACT}

Background and study aims Dietary restrictions are integral to colonoscopy preparation and impact patient satisfaction. Utilizing split-dose, lower-volume polyethylene glycol 3350-electrolyte solution (PEG-ELS), this study compared colon preparation adequacy of a low-residue diet to clear liquids using a validated grading scale. Patients and methods This was a prospective, randomized, singleblinded, single-center non-inferiority study evaluating diet the day prior to outpatient colonoscopy. Subjects were randomized to a Low-Residue diet for breakfast and lunch, or Clears only. All subjects received split dose PEG-ELS. The primary endpoint was preparation adequacy using the Boston Bowel Preparation Scale (BBPS), with adequate defined as a score $>5$. Secondary endpoints included mean BBPS scores for the entire colon and individual segments, satisfaction, adverse events, polyp and adenoma detection rates, and impact on sleep and daily activities.

Results Final analysis included 140 subjects, 72 assigned to Clears and 68 to Low-Residue. The Low-Residue diet was non-inferior to Clears (risk difference $=-5.08 \%, P=0.04$ ) after adjusting for age . Mean colon cleansing scores were not significantly different overall and for individual colonic segments. Satisfaction with the Low-Residue diet was significantly greater $(P=0.01)$. The adenoma detection rate was not statistically significantly different between study groups, but the number of adenomas detected was significantly greater with Clears $(P=0.01)$. Adverse events and impact on sleep and activities did not differ significantly between diet arms.

Conclusions A low-residue diet for breakfast and lunch the day prior to colonoscopy was non-inferior to clear liquids alone for achieving adequate colon cleansing when using split dose PEG-ELS.

\section{Introduction}

The process of preparing for colonoscopy is an important barrier to screening [1]. The side effects associated with purgative ingestion are compounded by dietary restrictions. FDA-approved purgatives have specific guidelines regarding diet, but for popular over-the-counter purgatives there are no specific recommendations. Irrespective of the purgative chosen, clinical practice may vary considerably with respect to the diet recommended prior to colonoscopy.

Many physicians recommend clear liquids the entire day prior to colonoscopy. Besides affecting patient satisfaction and willingness to undergo colonoscopy [1], such restriction may interfere with medical regimens (i.e. diabetes management), performance of daily activities, and attendance as well as productivity at work.

Regimens in which at least part of a purgative is dosed on the day of colonoscopy are associated with superior bowel cleansing and have been endorsed within published guidelines [2-5]. As greater acceptance of more effective regimens grows, liberalization of pre-procedure diets may be possible without sacrificing preparation adequacy. As compared to clear liquids alone, similar bowel cleansing scores have been demonstrated with a low-residue diet in conjunction with hyperosmotic bowel purgatives [6-8].

Polyethylene glycol-electrolyte solution (PEG-ELS) is an isoosmotic purgative commonly prescribed for colonoscopy. PEGELS has a good safety profile and few contraindications, and is the purgative of choice for vulnerable populations such as those with advanced cardiac, liver, or renal disease. In patients receiving split dose PEG-ELS for colonoscopy, our aim was to evaluate whether adequate colon cleansing may be achieved when a low-residue diet is consumed the day prior to colonoscopy. We hypothesized that a low-residue diet would be non-inferior to clear liquids for achieving an adequate preparation. 


\section{Patients and methods}

This was a prospective, randomized, single-blinded, single-center study evaluating the effect of diet on bowel preparation adequacy. All colonoscopies were performed by board-certified gastroenterologists without Fellow involvement. All subjects received monitored anesthesia care using propofol-based sedation administered by a certified registered nurse anesthetist. The study was approved by the Institution Review Board at Thomas Jefferson University and registered through the U.S. National Institutes of Health at ClincalTrials.gov (\#NCT01876576).

\section{Subjects}

Eligible subjects were $\geq 18$ years old who were scheduled to undergo elective outpatient colonoscopy. Exclusion criteria included pregnancy, breast feeding, gastroparesis (suspected or established), chronic nausea or vomiting, hypomotility syndromes (pseudo-obstruction, etc.), colonic resection, bowel obstruction, severe constipation $(\leq 1$ bowel movement per week), glucose-6-phosphate dehydrogenase deficiency, PEG allergy, significant psychiatry illness (schizophrenia, active bi-polar, severe depression, etc.), and inability or unwillingness to consent. Subjects could withdraw at any time.

\section{Consent and randomization}

Informed consent was provided in private by an investigator uninvolved in performing colonoscopy. Following consent, the same investigator immediately randomized the subject using a schedule generated by http://www.randomization.com. This investigator was not privy to the randomization assignment at the time of consent. Upon randomization, written instructions were reviewed with, and provided to, subjects regarding their specific assignment to a clear liquid diet ("Clears") or a low-residue diet ("Low-residue"). Subjects were instructed to not discuss their assigned diet with the physician performing the colonoscopy.

\section{Protocol}

The Clears Diet consisted of clear liquids for the entire day prior to colonoscopy and up to 2.5 hours before colonoscopy. Patients were provided with a list of permissible clear liquids. Patients in the Low-residue arm were permitted a low-residue diet the day prior to colonoscopy up to $1 \mathrm{pm}$, and this was followed by clear liquids up to 2.5 hours before colonoscopy. Patients created their own low-residue diet guided by an instruction sheet created by a registered dietician of examples of foods that are permissible and those that are not. (Addendum A)

All subjects in both study arms received 2 L PEG-ELS (MoviPrep ${ }^{\circledR}$, Salix Pharmaceuticals, Morrisville, NC) administered as a split dose. The first liter was consumed as 250 cc every 15 minutes, along with $500 \mathrm{cc}$ of clear liquids, beginning at $5 \mathrm{pm}$ the day prior to colonoscopy. This was repeated for the second liter beginning 4 hours prior to colonoscopy.

On the day of the procedure, immediately prior to colonoscopy, vital signs were measured (weight and orthostatic assessment of blood pressure and pulse) and questionnaires were administered assessing compliance, side effects, satisfaction, sleep quantity and quality, and ability to work as well as productivity the day prior to colonoscopy. Adverse events (AEs) were measured using a 10-point Likert scale from 0 (none) to 10 (severe). Sleep quantity was measured by comparing the average number of hours the subject normally sleeps to the number of hours they slept the night before colonoscopy. Sleep quality was rated on a 5-point scale: very poor (1), poor, average, good, and very good (5). Finally, work and productivity were measured using questions adapted from The Health and Labour Questionnaire [9]. (Addendum B)

Data recorded during the colonoscopy included procedure duration (total, insertion, and withdrawal excluding interventions) and cecal intubation, as well as polyp detection including number, location, size, and method of removal. Each polyp was placed in a separate container for pathology. Once available, final pathology results were recorded including histology (hyperplasia, adenoma, sessile serrated polyp, cancer, other), degree of dysplasia (low- or high-grade), and whether a villous component was present.

Immediately after completing the colonoscopy, the endoscopist evaluated the preparation using the Boston Bowel Preparation Scale (BBPS) [10]. Prior to beginning the study, each colonoscopist reviewed the BBPS training video made available by the Boston University School of Medicine (http://www.cori.org/ bbps). The endoscopist also estimated the amount of intra-procedure flushing (none, $<50 \mathrm{cc}, 50-100 \mathrm{cc},>100 \mathrm{cc}$ ) and indicated whether they remained blinded to the subject's diet assignment. If the colonoscopy preparation was poor, the endoscopist used personal judgment regarding whether the colonoscopy should be aborted.

\section{Endpoints}

The primary trial endpoint was the dichotomous index of adequate colon preparation (adequate [BBPS $>5$ ] versus inadequate $[B B P S \leq 5]$ ) for the whole colon. Secondary endpoints included BBPS scores for each graded segment (right, transverse, and left), polyp and adenoma detection rate (number of patients with at least one lesion/number of colonoscopies $X$ $100)$, side effects, preparation completion ( $\geq 90 \%$ consumed), quality of life measures (satisfaction, sleep quality, and day prior work and productivity), and other colonoscopy measures (flushing, total and withdrawal procedure duration, and cecal intubation).

\section{Statistics}

The primary endpoint was tested by a one-sided Wald test for non-inferior proportion of adequate preparation by the Low-residue diet as compared to Clears with a pre-specified margin. We expected about $90 \%$ of subjects assigned to Clears to be adequate, and the non-inferiority margin was set at a risk difference (RD) of $-13.5 \%$. The non-inferiority margin selected was based on the risk difference of several published purgative studies [11-15]. If the true adequacy of the comparators was indeed the same, then 70 subjects in each group was expected to provide $81 \%$ power to establish non-inferiority with a $5 \%$ type I error rate. 
For secondary analyses, summary statistics were computed for both groups on each endpoint along with two-sided superiority test statistic $P$ values. For the dichotomous endpoints, Fisher's exact test was used. Group differences across the 4 flushing categories were evaluated by the parametric Pearson's chi square test. Count data for the number of polyps and adenomas (included sessile serrated polyps) detected were evaluated by using generalized linear zero-inflated Poisson regression models to compute incidence rate ratios (IRR) between groups with $95 \%$ confidence intervals $(\mathrm{Cl})$. Group differences between continuous and ordinal variables were evaluated by Wilcoxon rank-sum test. Multivariable analyses were conducted to adjust for potential confounding for age, gender, and indication.

Descriptive statistics were used to describe the groups and uncover possible confounding of the Low-residue vs. Clears comparison for the primary endpoint. We adjusted the estimated RD for potential confounders by modeling them as covariates in a generalized linear binomial regression model. The zero-inflated Poisson models were also expanded to include adjustment terms for potential confounders. Sensitivity analyses for the number of adenomas detected were also conducted to determine the influence of adenoma multiplicity.

All tests were conducted with significance level $\alpha=0.05$. All analyses were performed on an intention to treat basis - all patients were included in the group to which they were randomized irrespective of whether they complied with the assigned diet or purgative. However, patients who withdrew consent or cancelled their colonoscopy were excluded from all analyses. Separate per protocol analyses are not provided as we felt that compliance with the assigned intervention or control treatment was similar across both groups, which would essentially make the per protocol and intention to treat analyses the same. The analyses were carried out with SAS 9.3 (SAS Institute Inc., Cary, NC).

\section{Results}

Between July 2012 and February 2013, a total of 230 patients were approached and 188 were randomized to either Clears ( $n$ $=94)$ or Low-residue $(n=94)$. A consort diagram summarizes the patient flow and disposition ( $\triangleright$ Fig. 1). Twenty-two patients assigned to Clears and 26 patients assigned to Low-residue withdrew from the study. The final analyses included a total of 140 patients with 72 assigned to Clears and 68 to Low-residue.

Patient characteristics are summarized in $>$ Table 1 . The Low-residue group had more women (61.8\% vs. $52.8 \%$ ), a greater proportion of older patients, and differences in indication for colonoscopy with more performed for symptoms $(23.9 \%$ vs. $13.9 \%)$ and fewer for surveillance $(28.4 \%$ vs. $38.9 \%$ ). Both age and gender were evaluated as possible confounders since they were both imbalanced between groups. However, unlike age, gender was not strongly related to the outcomes that were evaluated.

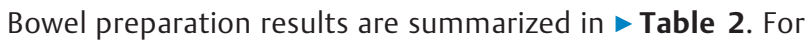
the primary outcome of preparation adequacy, the Low-residue diet was non-inferior to Clears $(R D=-5.08 \%, P=0.04)$ after ad-

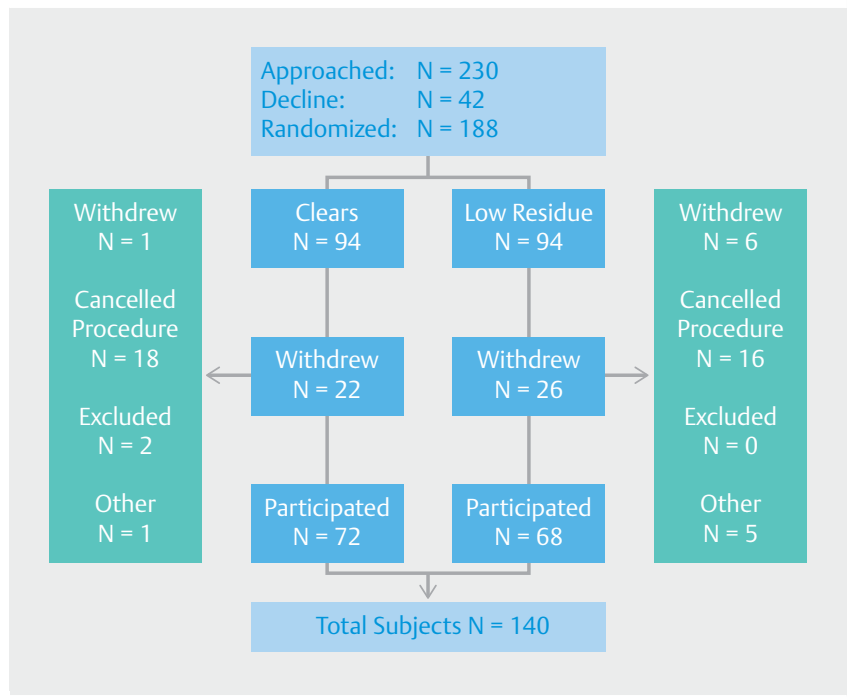

Fig. 1 Patient assignment and disposition flow diagram.

- Table 1 Subject characteristics.

\begin{tabular}{|l|l|l|l|}
\hline \multirow{2}{*}{ Characteristics, $\mathbf{n}$} & Clears (n=72) & $\begin{array}{l}\text { Low-residue } \\
\mathbf{( n = 6 8 )}\end{array}$ \\
\hline \multirow{2}{*}{ Gender } & Male & $34(47.2 \%)$ & $26(38.2 \%)$ \\
\cline { 2 - 4 } & Female & $38(52.8 \%)$ & $42(61.8 \%)$ \\
\hline \multirow{2}{*}{ Age } & $18-50$ & $30(41.7 \%)$ & $17(25.0 \%)$ \\
\hline \multirow{3}{*}{ Indication } & $51-60$ & $20(27.8 \%)$ & $26(38.2 \%)$ \\
\hline & Surveillance & $28(38.9 \%)$ & $19(28.4 \%)$ \\
\hline & Symptom & $10(13.9 \%)$ & $16(23.9 \%)$ \\
\hline
\end{tabular}

- Table 2 Bowel preparation.

\begin{tabular}{|c|c|c|c|}
\hline $\begin{array}{l}\text { Preparation } \\
\text { adequacy }{ }^{1} \text {, } \\
\text { n (\%) }(\text { BBPS }>5)\end{array}$ & $\begin{array}{l}\text { Clears } \\
(n=72)\end{array}$ & $\begin{array}{l}\text { Low-residue } \\
(n=68)\end{array}$ & $P$ value \\
\hline Yes & $68(94.4 \%)$ & $60(88.2 \%)$ & \multirow{2}{*}{0.04} \\
\hline No & $4(5.6 \%)$ & $8(11.8 \%)$ & \\
\hline \multicolumn{4}{|l|}{$\begin{array}{l}\text { BBPS Scores², } \\
\text { Mean (SD) }\end{array}$} \\
\hline Total & $7.61(1.5)$ & $7.15(1.93)$ & 0.22 \\
\hline Right & $2.46(0.63)$ & $2.44(0.66)$ & 0.33 \\
\hline Transverse & $2.57(0.58)$ & $2.45(0.68)$ & 0.93 \\
\hline Left & $2.56(0.60)$ & $2.37(0.67)$ & 0.08 \\
\hline \multicolumn{4}{|c|}{$\begin{array}{l}1 \text { One-sided non-inferiority test } \mathrm{p} \text {-value based on a binomial model of the } \\
\text { risk difference estimate adjusted for confounding from age. } \\
{ }^{2} \text { Superiority test } P \text { values. }\end{array}$} \\
\hline
\end{tabular}


justing for confounding associated with the imbalanced age distribution noted above. With respect to bowel preparation scores for the total colon and for each colonic segment (right, transverse, and left), there were no significant differences between the two study groups.

Compliance and satisfaction with diet assignment and purgative ingestion is summarized in $>$ Table 3 . Satisfaction with diet was significantly greater with a low-residue diet $(P=0.01)$. Adherence to diet instructions was similar between the study groups. While completion of greater than $90 \%$ of the evening dose of purgative was very similar among those assigned Clears vs. Low-residue (respectively, 92.9\% vs. 92.7\%), fewer patients in the Low-residue arm tended to complete greater than $90 \%$ of the morning purgative dose ( $91 \%$ vs. $79.1 \%, P=0.09$ ).

- Table 4 summarizes colonoscopy findings. The binary, patient-level adenoma detection rate was similar between the study groups (Low-residue vs. Clears: $35.3 \%$ vs. $44.4 \%$; $P=$ $0.30)$. However, the total number of adenomas detected was significantly greater in those receiving Clears (38 vs. $72, \mathrm{IRR}=$ 1.84 (95\% Cl: 1.16, 2.91); $P=0.01$ ). The binary, patient-level polyp detection rate was also similar in both groups, but the total number of polyps detected was also significantly greater in the Clears group (81 vs. 134, IRR 1.43 (95\% Cl: $1.04,1.96$ ); $P=$ $0.03)$. A sensitivity analysis where we removed the three patients with a large number of adenomas ( $>6$; each was assigned to the Clears group) was performed. This analysis found the difference in the total number of adenomas and polyps detected in the Clears group to no longer be significant (IRR =1.40, (95 $\% \mathrm{Cl}: 0.86,2.27) ; P=0.17$ and $\mathrm{IRR}=1.24$, (95\% Cl: 0.90, 1.72); $P=0.19)$. In terms of polyp size, an ordinal logistic regression model to this endpoint found that the odds of having a higher maximum polyp size did not depend significantly by diet $(\mathrm{OR}=$ $0.77 ; 95 \% \mathrm{Cl}: 0.34,1.74 ; P=0.53)$. $>$ Table 5 provides the number of patients within each study group according to three categories of polyp size.

There was no statistically significant difference between the study groups with respect to intra-procedure flushing and colo- noscopy duration (total, insertion, and withdrawal independent of interventions). Multivariable analyses revealed that age, gender, and indication were not significant predictors of cecal intubation or adenoma or polyp detection and did not confound the results shown for the comparison between study groups for these 2 endpoints.

Patients were not excluded on basis of taking medications that would slow gastrointestinal motility. Patients were asked to provide a list of their current medications. Table 6 summarizes the number and percentage of patients within each study group who were taking any one of eighteen recorded medication classes. There were no significant differences between the groups with respect to any of these 18 drug classes. There was a trend toward a higher percentage of the Clears group taking diabetes, psychiatric, or neurologic meds. There was also a trend toward a higher percentage of the Low-residue group taking vitamins, herbs, and supplements. The study groups did not significantly differ with respect to adverse events (abdominal pain, bloating, hunger, light headedness, nausea or vomiting), quality of sleep, or productivity the day prior to colonoscopy.

\section{Discussion}

It is now widely recognized that administering at least part of a purgative on the day of colonoscopy improves cleansing efficacy [16]. A secondary benefit of split dose regimens is improved patient satisfaction [8]. Our study addresses another source of patient dissatisfaction with colonoscopy: the diet preceding this procedure. Joint guidelines' discussion of diet for colonoscopy state "patients generally adopt a liquid diet one or more days before the examination" [17]. In addition to a full day or more of clear liquids, some physicians may recommend additional dietary alterations and/or other supplements to the prescribed purgative.

We found that a low-residue diet for breakfast and lunch the day prior to colonoscopy was non-inferior to clear liquids for

- Table 3 Subject compliance and satisfaction.

\begin{tabular}{|c|c|c|c|c|}
\hline Compliance, n (\%) & & $\begin{array}{l}\text { Clears } \\
(n=72)\end{array}$ & Low-residue $(n=68)$ & $P$ value \\
\hline \multirow[t]{2}{*}{ Diet } & Yes & $67(93.1 \%)$ & $59(86.8 \%)$ & \multirow{2}{*}{0.27} \\
\hline & No & $5(6.9 \%)$ & $9(13.2 \%)$ & \\
\hline \multirow[t]{2}{*}{ PM preparation completion ${ }^{1}$} & $>90 \%$ & $65(92.9 \%)$ & $63(92.7 \%)$ & \multirow{2}{*}{0.99} \\
\hline & $\leq 90 \%$ & $5(7.1 \%)$ & $5(7.4 \%)$ & \\
\hline \multirow[t]{2}{*}{ AM preparation completion ${ }^{1}$} & $>90 \%$ & $61(91 \%)$ & $53(79.1 \%)$ & \multirow{2}{*}{0.09} \\
\hline & $\leq 90 \%$ & $6(9 \%)$ & $14(20.9 \%)$ & \\
\hline \multicolumn{5}{|l|}{ Satisfaction, mean (SD) ${ }^{2}$} \\
\hline Bowel preparation & & $3.35(2.46)$ & $2.78(2.42)$ & 0.15 \\
\hline Diet & & $3.04(2.39)$ & $1.99(1.93)$ & 0.01 \\
\hline
\end{tabular}


Table4 Colonoscopy quality measures.

\begin{tabular}{|c|c|c|c|c|c|}
\hline \multicolumn{2}{|l|}{ Quality measures } & $\begin{array}{l}\text { Clears } \\
(n=72)\end{array}$ & Low-residue $(n=68)$ & $P$ value & IRR $^{1}$ (95\% Cl) \\
\hline \multirow{2}{*}{ Polyps } & $\geq 1$ polyp, $\mathrm{N}(\%)$ & 50 (69.4\%) & $37(54.4 \%)$ & 0.08 & \\
\hline & Total \# & 134 & 81 & 0.03 & $1.43(1.04,1.96)$ \\
\hline \multirow{2}{*}{ Adenomas } & zadenoma, n (\%) & $32(44.4 \%)$ & $24(35.3 \%)$ & 0.30 & \\
\hline & Total \# & 72 & 38 & 0.01 & $1.84(1.16,2.91)$ \\
\hline \multirow{2}{*}{ Cecal intubation n (\%) } & Yes & $69(95.8 \%)$ & $61(89.7 \%)$ & \multirow{2}{*}{0.19} & \\
\hline & No & $3(4.2 \%)$ & $7(10.3 \%)$ & & \\
\hline \multirow{3}{*}{ Duration (mean minutes and SD) } & Insertion & $6.72(3.28)$ & $7.37(3.93)$ & 0.35 & \\
\hline & Withdrawal ${ }^{2}$ & $7.92(3.27)$ & $7.32(2.33)$ & 0.73 & \\
\hline & Total & $14.82(4.89)$ & $14.88(4.6)$ & 0.86 & \\
\hline
\end{tabular}

- Table 5 Polyp size found within each diet group.

\begin{tabular}{|l|l|l|}
\hline Polyp size & Clears $\mathbf{n}(\%)$ & Low-residue $\mathbf{n}(\%)$ \\
\hline$<5 \mathrm{~mm}$ & $16(34.0)$ & $15(42.9)$ \\
\hline $5 \mathrm{~mm}$ to $<10 \mathrm{~mm}$ & $19(40.5)$ & $13(37.1)$ \\
\hline$\geq 10 \mathrm{~mm}$ & $12(25.5)$ & $7(20.0)$ \\
\hline
\end{tabular}

achieving an adequate colon preparation. A low-residue diet was non-inferior despite a lower rate of purgative completion on the day of colonoscopy. As a secondary outcome, mean bowel preparation scores for the whole colon, and by colon segment, were evaluated and found to be similar between both diets. While satisfaction with diet was superior in the low-residue group, no advantage was shown with respect to AEs, productivity, and quality measures (ADR, colonoscopy completion, withdrawal time). A significantly greater number of adenomas were detected in the Clears arm. Our sensitivity analyses of adenomas and polyps detected suggests that the significance of these findings were highly sensitive to possible outlier effects caused by a few patients assigned to the Clears having 6 or more adenomas detected. However, the IRRs from these sensitivity analyses were still high, suggesting that this study was underpowered for adenoma and polyp detection.

Rather than following a standardized or pre-packaged menu, each patient assigned to the low-residue diet was permitted to create their own menu based on guidelines created by a registered dietician. This approach has several advantages. To begin, it permits generalized adoption of this diet option outside a study setting. Furthermore, giving patients greater latitude permits adaptation to a variety of dietary needs and preferences. Finally, patients incur no additional charges that may be associated with pre-prepared meals.

Our study utilized a low-volume PEG-ELS purgative and builds on the work of others. A few studies have looked at the effect of diet in patients receiving hyperosmotic purgatives.
One study using split dose sodium phosphate liquid compared a low-residue breakfast to a low-residue breakfast and lunch. Using an unvalidated grading scale, these investigators found both diets to be efficacious [7]. Sipe et al compared clear liquids to a low-residue diet using split dose oral sulfate solution and found no significant difference in mean cleansing scores between the 2 groups [8]. Preparation adequacy was not an endpoint, and those assigned to the low-residue arm were required to strictly adhere to one of 3 specific menu offerings. A third study compared a low-residue diet to clears alone and used sodium picosulfate plus magnesium [6]. All patients received bisacodyl for 2 days prior to beginning the purgative. A hybrid dosing schedule was used: day prior for procedures before 11:30 am, and split dose for those with a later start time. The primary endpoint, mean cleansing scores, was not significantly different between the 2 arms. This study also graded cleansing with the Aronchick scale and found over $25 \%$ in the low-residue arm to have inadequate cleansing (using a standard definition where adequate is comprised of good and excellent preparations).

Studies focused on diet that have utilized PEG-ELS have been limited by problems with study design. One protocol varied both purgative and diet between study arms, comparing a prepackaged low-residue diet with picosulfate plus magnesium and bisacodyl to a "standard" diet with 4L PEG-ELS and bisacodyl [18]. Adequate bowel cleansing was superior in the low-residue arm. In another study, patients ingested $4 \mathrm{~L}$ PEG-ELS on the morning of colonoscopy and all patients followed a low-residue diet starting 3 days before colonoscopy [19]. However, on the day prior to colonoscopy 1 group continued this while the other group followed a clear liquid diet. In this study, a clear liquid diet was not found to be superior to a low-residue diet.

Despite low-residue food up to $1 \mathrm{pm}$, and starting the purgative at $5 \mathrm{pm}$, we did not observe greater productivity the day prior to colonoscopy in the Low-residue subjects. A prior study evaluating the efficacy of purgative administration entirely on the day of colonoscopy also found no significant improvement 
- Table 6 Classes of medications used in each diet group.

\begin{tabular}{|c|c|c|c|}
\hline Type of medication & Clears n (\%) & Low-residue n (\%) & $P$ value $^{1}$ \\
\hline Antihypertensive & & & 0.73 \\
\hline No & $44(61.1 \%)$ & $44(64.7 \%)$ & \\
\hline Yes & $28(38.9 \%)$ & $24(35.3 \%)$ & \\
\hline Diabetes & & & 0.08 \\
\hline No & $62(86.1 \%)$ & $65(95.6 \%)$ & \\
\hline Yes & $10(13.9 \%)$ & $3(4.4 \%)$ & \\
\hline IBD & & & 0.39 \\
\hline No & $67(93.1 \%)$ & $60(88.2 \%)$ & \\
\hline Yes & $5(6.9 \%)$ & $8(11.8 \%)$ & \\
\hline Acid suppression & & & 0.58 \\
\hline No & $48(66.7 \%)$ & $49(72.1 \%)$ & \\
\hline Yes & $24(33.3 \%)$ & $19(27.9 \%)$ & \\
\hline Psychiatric & & & 0.10 \\
\hline No & $53(73.6 \%)$ & $58(85.3 \%)$ & \\
\hline Yes & $19(26.4 \%)$ & $10(14.7 \%)$ & \\
\hline Cardiac & & & 0.28 \\
\hline No & $45(62.5 \%)$ & $49(72.1 \%)$ & \\
\hline Yes & $27(37.5 \%)$ & $19(27.9 \%)$ & \\
\hline Allergy & & & 0.55 \\
\hline No & $67(93.1 \%)$ & $61(89.7 \%)$ & \\
\hline Yes & $5(6.9 \%)$ & $7(10.3 \%)$ & \\
\hline Asthma/COPD & & & 0.74 \\
\hline No & $68(93.1 \%)$ & $61(89.7 \%)$ & \\
\hline Yes & $4(5.6 \%)$ & $7(10.3 \%)$ & \\
\hline Neurologic & & & 0.17 \\
\hline No & $65(90.3 \%)$ & $66(97.1 \%)$ & \\
\hline Yes & $7(9.7 \%)$ & $2(2.9 \%)$ & \\
\hline Antibiotics & & & - \\
\hline No & $72(100 \%)$ & $68(100 \%)$ & \\
\hline Laxatives & & & 1.00 \\
\hline No & 71 (98.6\%) & $67(98.5 \%)$ & \\
\hline Yes & $1(1.4 \%)$ & $1(1.5 \%)$ & \\
\hline Aspirin/NSAIDs & & & 0.60 \\
\hline No & $65(90.3 \%)$ & $59(86.6 \%)$ & \\
\hline Yes & $7(9.7 \%)$ & $9(13.2 \%)$ & \\
\hline Renal & & & - \\
\hline No & $72(100.0 \%)$ & $68(100.0 \%)$ & \\
\hline Thyroid & & & 1.00 \\
\hline
\end{tabular}


- Table 6 (Continuation)

\begin{tabular}{|l|l|l|}
\hline Type of medication & Clears $\mathbf{n}(\%)$ & Low-residue $\mathbf{n}(\%)$ \\
\hline No & $65(90.3 \%)$ & $61(89.7 \%)$ \\
\hline Yes & $7(9.7 \%)$ & $7(10.3 \%)$ \\
\hline Vitamins/herbals/supplements & & 0.14 \\
\hline No & $61(84.7 \%)$ & $50(73.5 \%)$ \\
\hline Yes & $11(15.3 \%)$ & $18(26.5 \%)$ \\
\hline Oncologic & & \\
\hline No & $72(100.0 \%)$ & $68(100.0 \%)$ \\
\hline Steroids/immunosuppressants & & $66(97.1 \%)$ \\
\hline No & $72(100.0 \%)$ & $2(2.9 \%)$ \\
\hline Yes & 0 & 0.23 \\
\hline Anticholinergics \& antidiarrheals & & $67(98.5 \%)$ \\
\hline No & $71(98.6 \%)$ & $1(1.5 \%)$ \\
\hline Yes & $1(1.4 \%)$ & 1.00 \\
\hline $\begin{array}{l}\text { COPD, chronic obstructive pulmonary disease; NSAID, nonsteroidal anti-inflammatory drug } \\
1 P \text { value from Fisher/s exact test. }\end{array}$ & \\
\hline
\end{tabular}

in productivity or attendance at work the day prior (though those attending work had less interference with the work day with AM dosing) when compared to split dosing. We speculate that patient anticipation of problems with the preparation process may play a role here, and that better education at the time of scheduling might lessen the impact on productivity and attendance at work the day prior to colonoscopy.

Our study has some limitations. To begin, there was a high drop-out rate in both arms, which can be attributed to financial limitations and expected withdrawal. As an unfunded study, there was limited research personnel time, necessitating restriction of the subject's colonoscopy date and time preferences. Subjects who rescheduled or cancelled procedures on short notice could no longer participate in our study. The lowresidue diet was non-inferior to clears, but this does not establish equivalence of the 2 diets or appropriateness for all patients undergoing colonoscopy. Additionally, analysis of this endpoint required adjustment for age imbalance between the groups. We assume this imbalance was due to random chance, although it is not clear if age might have interacted with diet assignment to influence the likelihood of withdrawal from the study. While not statistically greater, failure to achieve cecal intubation occurred more frequently in the Low-residue arm and this is likely related to inadequate cleansing. Excluded from this study were severely constipated patients ( $\leq 1$ bowel movement per week) and those with known hypomotility disorders.

Medications known to slow gastrointestinal transit or increase the risk for inadequate colon cleansing were not exclusion criterion. There was no significant difference between the study groups with respect to the use of any of 18 recorded medication classes. However, there was a non-statistically sig- nificant trend toward greater use of diabetic, neurological, and psychiatric medications in the Clears group, which could have negatively influenced preparation scores in this group. Also, while we observed no instances of endoscopist unblinding to diet regimen, this possibility and its impact cannot be completely excluded. Furthermore, ours is a single-center study and the results may not be generalizable. Lastly, significantly more polyps and adenomas were detected in the Clears group. As mentioned above, this study was underpowered for detecting differences in the rates of adenoma and polyp detection. This study cannot evaluate whether the actual polyp and adenoma counts in these groups were disparate by chance or related to diet assignment.

\section{Conclusions}

In conclusion, a low volume PEG-ELS purgative administered as a split dose with a low-residue breakfast and lunch the day prior to colonoscopy improves patient satisfaction and is not inferior to clear liquids for achieving adequate bowel cleansing for colonoscopy. Adoption of this diet regimen may help breakdown one of the existing barriers to colonoscopy and should be considered, particularly for those who are low risk for preparation failure. A much larger study evaluating differences between these diet regimens for quality measures such as cecal intubation and adenoma detection would provide valuable information. 
Competing interests

None

References

[1] Harewood GC, Wiersema M], Melton LJ3rd et al. A prospective, controlled assessment of factors influencing acceptance of screening colonoscopy. Am J Gastroenterol 2002; 97: 3186-3194

[2] Soweid AM, Kobeissy AA, Jamali FR et al. A randomized single-blind trial of standard diet versus fiber-free diet with polyethylene glycol electrolyte solution for colonoscopy preparation. Endoscopy 2010; 42: $633-638$

[3] Gurudu SR, Ramirez FC, Harrison ME et al. Increased adenoma detection rate with system-wide implementation of a split-dose preparation for colonoscopy. Gastrointest Endosc 2012; 76: 603-608 e601

[4] Mamula P, Adler DG, Conway JD et al. Colonoscopy preparation. Gastrointest Endosc 2009; 69: 1201 - 1209

[5] Rex DK, Johnson DA, Anderson JC et al. American College of Gastroenterology guidelines for colorectal cancer screening 2009 [corrected]. Am J Gastroenterol 2009; 104: 739 - 750

[6] Melicharkova A, Flemming J, Vanner $S$ et al. A low-residue breakfast improves patient tolerance without impacting quality of low-volume colon cleansing prior to colonoscopy: a randomized trial. Am J Gastroenterol 2013; 108: 1551-1555

[7] Scott SR, Raymond PL, Thompson WO et al. Efficacy and tolerance of sodium phosphates oral solution after diet liberalization. Gastroenterol Nurs 2005; 28: $133-139$

[8] Sipe BW, Fischer M, Baluyut AR et al. A low-residue diet improved patient satisfaction with split-dose oral sulfate solution without impairing colonic preparation. Gastrointest Endosc 2013; 77: 932 - 936

[9] van Roijen L, Essink-Bot ML, Koopmanschap MA et al. Labor and health status in economic evaluation of health care. The Health and Labor Questionnaire. . Int J Technol Assess Health Care 1996; 12: 405-415

[10] Lai E], Calderwood AH, Doros G et al. The Boston bowel preparation scale: a valid and reliable instrument for colonoscopy-oriented research. Gastrointest Endosc 2009; 69: 620-625
[11] Bitoun A, Ponchon T, Barthet M et al. Results of a prospective randomised multicentre controlled trial comparing a new 2-L ascorbic acid plus polyethylene glycol and electrolyte solution vs. sodium phosphate solution in patients undergoing elective colonoscopy. Aliment Pharmacol Ther 2006; 24: 1631 -1642

[12] Rex DK, Schwartz H, Goldstein M et al. Safety and colon-cleansing efficacy of a new residue-free formulation of sodium phosphate tablets. Am J Gastroenterol 2006; 101: 2594-2604

[13] DiPalma JA, McGowan J, Cleveland MV. Clinical trial: an efficacy evaluation of reduced bisacodyl given as part of a polyethylene glycol electrolyte solution preparation prior to colonoscopy. Aliment Pharmacol Ther 2007; 26: $1113-1119$

[14] Matro R, Shnitser A, Spodik M et al. Efficacy of morning-only compared with split-dose polyethylene glycol electrolyte solution for afternoon colonoscopy: a randomized controlled single-blind study. Am J Gastroenterol 2010; 105: 1954-1961

[15] Di Palma JA, Rodriguez R, McGowan J et al. A randomized clinical study evaluating the safety and efficacy of a new, reduced-volume, oral sulfate colon-cleansing preparation for colonoscopy. Am J Gastroenterol 2009; 104: 2275-2284

[16] Aoun E, Abdul-Baki H, Azar C et al. A randomized single-blind trial of split-dose PEG-electrolyte solution without dietary restriction compared with whole dose PEG-electrolyte solution with dietary restriction for colonoscopy preparation. Gastrointest Endosc 2005; 62: $213-218$

[17] Levin B, Lieberman DA, McFarland B et al. Screening and surveillance for the early detection of colorectal cancer and adenomatous polyps, 2008: a joint guideline from the American Cancer Society, the US Multi-Society Task Force on Colorectal Cancer, and the American College of Radiology. Gastroenterology 2008; 134: 1570 - 1595

[18] Kim YS, Hong CW, Kim BC et al. Randomized clinical trial comparing reduced-volume oral picosulfate and a prepackaged low residue diet with 4-liter PEG solution for bowel preparation. Dis Colon Rectum 2014; 57: $522-528$

[19] Jung YS, Seok HS, Park DI et al. A clear liquid diet is not mandatory for polyethylene glycol-based bowel preparation for afternoon colonoscopy in healthy outpatients. Gut Liver 2013; 7: $681-687$ 